OPEN ACCESS

Edited by:

Eric Ruelland,

UMR7618 Institut

D'Écologie et des Sciences de

L'Environnement de Paris (IEES),

France

Reviewed by:

Severine Planchais,

Université Pierre et Marie Curie,

France

Jin Zhang,

Oak Ridge National Laboratory (DOE),

United States

*Correspondence:

Guohua Chai

chaigh@qau.edu.cn

Gongke Zhou

zhougk@qibebt.ac.cn

Specialty section:

This article was submitted to

Plant Abiotic Stress,

a section of the journal

Frontiers in Plant Science

Received: 02 August 2019 Accepted: 12 December 2019

Published: 24 January 2020

Citation:

Zhuang $Y$, Wang $C$, Zhang $Y$,

Chen S, Wang D, Liu Q, Zhou G and Chai $G$ (2020) Overexpression of

PdC3H17 Confers Tolerance to

Drought Stress Depending on

Its CCCH Domain in Populus.

Front. Plant Sci. 10:1748.

doi: 10.3389/fp/s.2019.01748

\section{Overexpression of $\mathrm{PdC} 3 \mathrm{H17}$ Confers Tolerance to Drought Stress Depending on Its $\mathrm{CCCH}$ Domain in Populus}

\author{
Yamei Zhuang ${ }^{1,2}$, Congpeng Wang ${ }^{3}$, Yang Zhang ${ }^{1,2}$, Sihui Chen ${ }^{2}$, Dian Wang ${ }^{2}$, Qing Liu $^{3}$, \\ Gongke Zhou $^{2 *}$ and Guohua Chai ${ }^{2,3 *}$ \\ 1 University of Chinese Academy of Sciences, Beijing, China, ${ }^{2}$ Key Laboratory of Biofuels, Chinese Academy of Sciences, \\ Shandong Provincial Key Laboratory of Energy Genetics, Qingdao Institute of Bioenergy and Bioprocess Technology, \\ Chinese Academy of Sciences, Qingdao, China, ${ }^{3}$ College of Resources and Environment, Qingdao Agricultural University, \\ Qingdao, China
}

Plant $\mathrm{CCCH}$ zinc finger proteins control growth, development, and stress responses mainly at the post-transcriptional level. Currently, limited reports are available about the roles of plant $\mathrm{CCCH}$ proteins in drought tolerance. In this study, we provided evidence showing that $P d \mathrm{C} 3 \mathrm{H} 17$ from Populus deltoides $\times P$. euramericana involves drought tolerance and response. Overexpression of $\mathrm{PdC} 3 \mathrm{H} 17$ in poplar caused dwarf, resulted in higher stem water potential, and showed increased photosynthetic and ROS-scavenging abilities, thereby enhancing tolerance to drought stress, compared to controls. Accordingly, after drought treatment the stem elongation and thickening rates of these overexpression lines were higher than those of the controls. However, overexpression of the coding region excluding the $\mathrm{CCCH}$ domain of $\mathrm{PdC} 3 \mathrm{H} 17$ roughly exhibited WT-like physiological and drought-resistant phenotypes, indicating the requirement of the $\mathrm{CCCH}$ domain for $\mathrm{PdC} 3 \mathrm{H} 17$ controlling these processes. In addition, $\mathrm{N}$-terminal sequence of $\mathrm{PdC} 3 \mathrm{H} 17$ was found to possess transcriptional activity ability in yeast cells. Together, our results suggest that $\mathrm{PdC} 3 \mathrm{H} 17$ may depend on its $\mathrm{CCCH}$ domain to control drought tolerance in Populus.

Keywords: the $\mathrm{CCCH}$ domain, $\mathrm{PdC} 3 \mathrm{H} 17$, drought tolerance, ROS scavenging activity, xylem vessel cell number, Populus

\section{INTRODUCTION}

Poplar (Populus spp.), a fast-growing tree species, is widely used for timber, pulp, and paper, and has potential as a source of bioenergy (Du and Groover, 2010). Poplars are drought-sensitive woody species and have evolved versatile mechanisms to mitigate drought stress, including reducing transpiration, scavenging reactive oxygen species (ROS), generating abscisic acid (ABA), and altering plant morphology (Harfouche et al., 2014). Accumulating evidence in Populus shows that a large amount of genes participate in controlling these processes under drought conditions. For example, $P d \mathrm{EPF} 1$, a member of the epidermal patterning factor (EPF) family in Populus nigra $\times$ (Populus deltoides $\times$ Populus nigra), regulates water use efficiency and drought tolerance by 
modulating stomatal density (Wang et al., 2016). PeCHYR1, an ubiquitin E3 ligase in Populus euphratica, enhances drought tolerance via ABA-induced stomatal closure by ROS production (He et al., 2018). The AREB1 transcription factor influences histone acetylation of PtrNAC006, PtrNAC007, and PtrNAC120, resulting in key physiological alterations conducive to drought tolerance and resilience and thereby changing drought responses in Populus trichocarpa (Li et al., 2019). Currently, it remains unclear that the regulatory mechanisms underlying drought response and tolerance in tree species.

The $\mathrm{CCCH}$ zinc finger family contains a typical C3H-type motif and members of this family had already been identified in organisms from yeast to human (Chai et al., 2015). Plant CCCH proteins play vital roles in a wide variety of growth, development, and stress responses, and may perform both transcriptional and posttranscriptional regulation (Bogamuwa and Jang, 2014). In Arabidopsis, the subfamily IX members of $\mathrm{CCCH}$ proteins are shown to mediate stress signaling based on qRT-PCR analysis (Wang et al., 2008). This is validated by subsequent functional analyses. AtTZF1/AtC3H23 functions as a regulator of ABA- and GA-mediated growth and drought response (Lin et al., 2011). Overexpression of AtTZF2/AtC3H20 or AtTZF3/AtC3H49, the homologs of AtTZF1, improves ABA hypersensitivity, reduces transpiration, and enhances drought tolerance (Lee et al., 2012). OsTZF1 is an ortholog of AtTZF1 in rice and confers tolerance to drought and high-salt stresses by regulating stress-related genes (Jan et al., 2013). OsC3H47 decreases ABA sensitivity and promotes drought and salt tolerance in rice seedlings (Wang et al., 2015). GhTZF1, an ortholog of AtTZF1 in cotton, regulates drought stress responses and delays leaf senescence by inhibiting ROS accumulation in transgenic Arabidopsis (Zhou et al., 2014). Recently, a non-tandem $\mathrm{CCCH}$ protein, $\mathrm{IbC} 3 \mathrm{H} 18$, is shown to act as a nuclear transcriptional activator and enhances salt, drought, and oxidation stress tolerance in sweet potato (Zhang et al., 2019), suggesting that in addition to subfamily IX, the members of other $\mathrm{CCCH}$ subfamilies in Arabidopsis may participate in controlling drought tolerance.

Our previous study demonstrated that Populus contains 91 CCCH gene family members, $90 \%$ of which are physically distributed on the duplicated blocks (Chai et al., 2012). Thirtyfour paralogous pairs are identified in these CCCHs, of which 22 pairs $(65 \%)$ may be created by the whole genome segment duplication. Of them, $P d \mathrm{C} 3 \mathrm{H} 17$ and $P d \mathrm{C} 3 \mathrm{H} 18$, a pair of paralogs, are direct targets of $P d \mathrm{MYB} 3$ and $P d \mathrm{MYB} 21$, which are second-level master switches of the transcription network for wood formation (Ye and Zhong, 2015), and function as positive regulators of secondary xylem development in both Arabidopsis and poplar (Chai et al., 2014). PdC3H17 has two orthologs (AtC3H14 and AtC3H15, belonging to subfamily II in CCCHs) in Arabidopsis (Wang et al., 2008; Chai et al., 2014). AtC3H14 and AtC3H15 redundantly control stem elongation and secondary cell wall thickening as well as anther development (Kim et al., 2014; Chai et al., 2015). However, the roles of the $\mathrm{CCCH}$ proteins in response to environmental stresses in trees have not yet been elucidated.

In this study, we provided evidence showing that $\mathrm{PdC} 3 \mathrm{H} 17$ controls drought response depending on its $\mathrm{CCCH}$ domain in a hybrid poplar. Overexpression of $\mathrm{PdC} 3 \mathrm{H} 17$ in poplar resulted in stronger drought-tolerance phenotype, correlating with a significant increase in both leaf ROS-scavenging abilities and stem xylem vessel cell numbers, compared to control plants. However, transgenic poplars overexpressing the coding region excluding the $\mathrm{CCCH}$ domain of $\mathrm{PdC} 3 \mathrm{H} 17$ roughly exhibited wild type-like phenotypes. Combined with other biochemical evidence, we suggest that $P d \mathrm{C} 3 \mathrm{H} 17$ may be a novel regulator of drought tolerance in poplar.

\section{MATERIALS AND METHODS}

\section{Transcriptional Activation Assay in Yeast}

The full-length coding region $(P d \mathrm{C} 3 \mathrm{H} 17)$ and the fragments including $(P d \mathrm{C} 3 \mathrm{H} 17 \Delta \mathrm{N})$ or excluding $(P d \mathrm{C} 3 \mathrm{H} 17 \Delta \mathrm{C})$ the $\mathrm{CCCH}$ domain of $P d C 3 H 17$ (Figure 1A) were separately fused in frame with the GAL4 DNA-binding domain in pGBKT7 (Clontech) using the appropriate primers containing the EcoRI site. PdC3H17: (Forward, 5'-ATGGAGGCCGAATTCATGGAG AAAACAGAATCACCA-3'; Reverse, 5'-GATCCCCGGGAAT TCTCAACGAGGACCCAGCAGTAACC-3'). PdC3H17DC: (Forward, 5'-ATGGAGGCCGAATTCATGGAGAAAACA GAATCACCA-3'; Reverse, 5'-GATCCCCGGGAATTCC

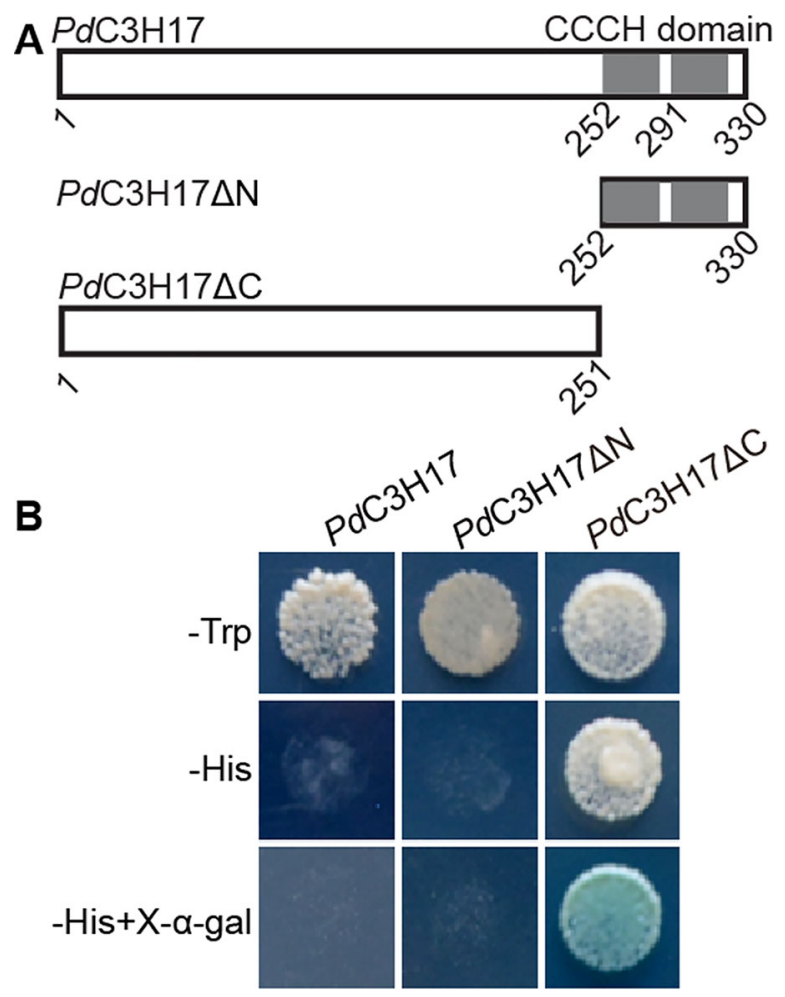

FIGURE 1 | Transactivation analysis of different regions of $\mathrm{PdC} 3 \mathrm{H} 17$ fused with the GAL4 DNA-binding domain in yeast. (A) Diagram of $P d C 3 H 17$, $P d C 3 H 17 \Delta N$, and $P d C 3 H 17 \Delta \mathrm{C}$ proteins. (B) Only $P d C 3 H 17 \Delta \mathrm{C}$ is able to activate the expression of the HIS3 reporter gene. 
GTGAAGgTtTTGGCGGGAA-3'). PdC3H17AN: (Forward, 5' -ATGGAGGCCGAATTCATGCAAGGGATGTGGAAG ACAG-3'; Reverse, 5'-GATCCCCGGGAATTCGTCAG TGAGGGAGTGGCGAAA- $3^{\prime}$ ). The recombinant vectors and the pGBKT7 empty vector were transformed into yeast strain AH109. The yeast liquid cultures were dropped on the synthetic dropout (SD)/Trp- and SD/Trp-/His-/Ade (adenine)-agar media. The transcriptional activation activity of each protein was evaluated according to their growth status and the activity of $\alpha$-galactosidase.

\section{Vector Construction and Plant Transformation}

Full-length $P d \mathrm{C} 3 \mathrm{H} 17$ and $P d \mathrm{C} 3 \mathrm{H} 17 \Delta \mathrm{C}$ were separately ligated downstream of the $35 \mathrm{~S}$ promoter in pCAMBIA1300-GFP vector to generate the overexpression constructs. Primers for $\mathrm{PdC} 3 \mathrm{H} 17$ were (Forward, 5'-CGGGGTACCATGGAGAAAACAGA ATCACCA-3', the KpnI site marked with underline) and (Reverse, 5' - CGGGA TCCTCAACGAGGACCCAGC AGTAACC- $3^{\prime}$, the BamHI site). Primers for $P d C 3 H 17 \Delta C$ were (Forward, 5'-CGGGGTACCATGGAGAAAACA GAATCACCA$3^{\prime}$, the KpnI site) and (Reverse, 5' CGGGATCCCGTGAAGGTTTTGGCGGAA- ${ }^{\prime}$, the BamHI site). The resulting construct was introduced into Populus deltoides $\times$ P. euramericana $\mathrm{cv}$ "nanlin895" via leaf disc method (Chai et al., 2014). The transgenic poplar plantlets were selected on a medium containing $5 \mathrm{mg} \mathrm{L}^{-1}$ hygromycin and identified through PCR at the DNA level and qRT-PCR at the mRNA level. PCR primers: 35Spro: ATGACGCACAATCCCACTATCC; PdC3H17R: 5' -CAGTGAGGGAGTGGCGAAAG-3'; PdC3H17ACR: 5'-TCGTTTGCATTAAACACCTCCA-3'. qRTPCR primers: PdC $3 \mathrm{H} 17$ (Forward: $5^{\prime}$ CAAGTGGCAAGAGACAGGCA-3'; Reverse: 5'-CAG TGAGGGAGTGGCGAAAG-3') and $P d \mathrm{C} 3 \mathrm{H} 17 \Delta \mathrm{C}$ (Forward: $5^{\prime}-$ ACTCAACTCGGTCTCGTGTG-3'; Reverse: 5'-TCGT TTGCATTAAACACCTCCA-3'). Regenerated plantlets were acclimatized in a mist chamber for 30 days, and transferred to a greenhouse with a $16 / 8 \mathrm{~h}$ light/dark photoperiod at $25^{\circ} \mathrm{C}$ to $28^{\circ} \mathrm{C}$.

\section{Quantitative Real-Time RT-PCR (qRT- PCR)}

Total RNA isolation and first-strand cDNA synthesis were performed as described previously (Chai et al., 2014). The qRTPCR assays were conducted on a LightCycler ${ }^{\circledR} 480$ Detection System (Roche) using a SYBR Premix Ex Taq II (TaKaRa) kit. The expression was normalized using reference gene $P d U B Q$ (BU879229, Forward, 5'-GTTGATTTTTGCTGGGAAGC-3'; Reverse, 5'-GATCTTGGCCTTCACGTTGT-3'), and determined by the $2^{-\Delta \Delta C T}$ method (Livak and Schmittgen, 2001). Data represent the average of at least three biological replicates.

\section{Microscopy}

Cross section of the basal stems was produced from 3-month-old transgenic poplars as described previously (Chai et al., 2014). For each construct, at least six plants of two lines were examined. Briefly, $0.5-\mathrm{cm}$ stem segments were submerged in $4 \%$ paraformaldehyde for 3 days, then dehydrated in a graded ethanol series, and finally incubated in pure paraplast. The paraplast-embedded stems were sectioned to a thickness of $7 \mu \mathrm{m}$ using a Leica RM 2235 microtome (Leica) and adhered to Superfrost Plus microscope slides (Thermo Fisher). Stem sections were stained with Toluidine Blue-O (TBO, 1\% w:v) and then observed using an Olympus DX51 microscope.

\section{Drought Treatment}

Three-month-old transgenic poplar lines encountered drought treatment, in which the soil RWC was reduced from $70 \%$ following described previously (Wang et al., 2016). Control plants were kept in the same conditions, except that the soil RWC was maintained at $70 \%$. Control and transgenic plants were grown in suitably sized pots, and each pot had a tray. Photographs were taken after treatment for 20 days.

\section{Measurement of Physiologic Parameters}

At 20th day of drought treatment, net photosynthetic rate, stomatal conductance, transpiration, and chlorophyll $\mathrm{a}+\mathrm{b}$ content were detected in the 5th to 7th leaves of control and transgenic lines using Li-6400 Photosynthesis System (Li-Cor Biosciences, Lincoln, NE). The 4th to 6th leaves from the top of the plants were sampled for measurement of the amounts of malondialdehyde (MDA), hydrogen peroxide $\left(\mathrm{H}_{2} \mathrm{O}_{2}\right)$, four ROSscavenging enzymes (superoxide dismutase, SOD; peroxidase, POD; ascorbate peroxidase, APX; and catalase, CAT) and three osmotic adjustments (proline, soluble protein, and soluble sugar). At least five biological replicates were performed for each genotype. The levels of $\mathrm{H}_{2} \mathrm{O}_{2}$, SOD, POD, APX, CAT, and soluble protein were measured according to the methods described by Shi et al. (2014).

The levels of MDA, proline, and soluble sugar were detected with the commercial kits following the instructions (Nanjing Jiancheng Bioengineering Institute, China). For determination of MDA content, $0.1 \mathrm{~g}$ of sample was extracted with $1 \mathrm{~mL}$ of $20 \%$ (w/v) trichloroacetic acid (TCA). The homogenate was centrifuged at $3500 \mathrm{~g}$ for $20 \mathrm{~min}, 2 \mathrm{~mL}$ supernatant was added to $2 \mathrm{~mL}$ of $20 \%$ TCA containing $0.5 \%(\mathrm{w} / \mathrm{v})$ triobarbituric acid (TBA). After heating in $95^{\circ} \mathrm{C}$ for $30 \mathrm{~min}$ and cooling in ice bath, the proline level was calculated based on absorbance at 532 and $600 \mathrm{~nm}$. The value for non-specific absorption at $600 \mathrm{~nm}$ was subtracted from the value at $532 \mathrm{~nm}$. For determination of proline content, $0.1 \mathrm{~g}$ of sample was ground and extracted in $3 \%(\mathrm{w} / \mathrm{v})$ sulphosalicylic acid. After reacting with acid ninhydrin solution, the proline level was calculated based on absorbance at $520 \mathrm{~nm}$. For the determination of soluble sugar content, $0.1 \mathrm{~g}$ of sample was extracted in $5 \mathrm{ml}$ of $80 \%(\mathrm{v} / \mathrm{v})$ ethanol at $80 \mathrm{C}$ for $40 \mathrm{~min}$ and centrifuged at $12000 \mathrm{rpm}$ for $10 \mathrm{~min}$. The supernatants were dipigmented by litter activated charcoal at $80^{\circ} \mathrm{C}$ for $30 \mathrm{~min}$. The mixture of $0.1 \mathrm{ml}$ of the extracts and $3 \mathrm{ml}$ of $0.15 \%(\mathrm{w} / \mathrm{v})$ anthrone reagent $(0.3 \mathrm{~g}$ anthrone was dissolved in $200 \mathrm{ml}$ of $7.74 \mathrm{M} \mathrm{H}_{2} \mathrm{SO} 4$ ) was heated at $90^{\circ} \mathrm{C}$ for $20 \mathrm{~min}$. The level of soluble sugar was examined at $620 \mathrm{~nm}$ of absorbance by making the specification curve with known concentration of glucose. 
Water potential was measured under well-watered condition or with drought treatment for 20 days. For each genotype, at least six plants of two lines were selected. A TP-PW-II Water Potential System (TPYN SciTech, Hangzhou, China) was used for the measurement of stem water potential according to the manufacturer's instructions. Statistical analyses were performed based on data from two independent experiments.

\section{Statistical Analysis}

All data were presented as mean \pm standard errors. The statistical significance of differences between data was evaluated using oneway analysis of variance (ANOVA) followed by Duncan's multiple range test $(P<0.05)$.

\section{RESULTS}

\section{Transcriptional Activation Analysis of PdC3H17}

Our previous study showed that Populus $\mathrm{PdC} 3 \mathrm{H} 17$ functions as positive regulators of secondary xylem development in both Arabidopsis and poplar (Chai et al., 2014). To elucidate the transcriptional properties of $P d \mathrm{C} 3 \mathrm{H} 17$, multiple fusion proteins were constructed, in which the coding region and selected portions of $P d \mathrm{C} 3 \mathrm{H} 17$ were fused to the GAL4 DNA-binding domain. All of the transformed yeast cells grew well on SD/Trp-medium. Transcription of the HIS reporter gene was significantly activated by GAL4- $P d \mathrm{C} 3 \mathrm{H} 17 \mathrm{~N}$-terminal region that excludes the $\mathrm{CCCH}$ domain $(\triangle \mathrm{C})$, but not by the GAL4-full-length $P d \mathrm{C} 3 \mathrm{H} 17$ protein $(\mathrm{FL})$ and the GAL4-CCCH domain $(\Delta \mathrm{N})$ (Figure 1). These results indicated that the $\mathrm{N}$-terminal sequence of $\mathrm{PdC} 3 \mathrm{H} 17$ may possess transcriptional activation capacity.

\section{Phenotypes of Transgenic Poplars Overexpressing PdC3H17 or PdC3H17 $\triangle \mathrm{C}$}

The structure and size of xylem vessel cells are key factors affecting water transport in plants and are important determinants of drought tolerance (Fisher et al., 2007; Li et al., 2019). To investigate whether $P d C 3 \mathrm{H} 17$ participates in controlling drought response, we generated transgenic poplar lines overexpressing the full length coding region $(P d \mathrm{C} 3 \mathrm{H} 17 \mathrm{OE})$ or the fragment excluding the $\mathrm{CCCH}$ domain $(P d \mathrm{C} 3 \mathrm{H} 17 \triangle \mathrm{COE})$ of $\mathrm{PdC} 3 \mathrm{H} 17$ (Figure 1A). For each construct, at least 30 independent transgenic lines were generated and two lines with highest expression of the transgene were selected for further characterization (Figures 2A, B). Consistent with our previous phenotypic observation (Chai et al., 2014), $P d$ C3H17OE lines exhibited dwarf compared with WT controls (Figure 2C). Considering that AtC3H14 and AtC3H15 redundantly control cell elongation in stems (Kim et al., 2014; Chai et al., 2015), we suggest that $P d C 3 H 17$ may play a negative role in controlling stem elongation. Interestingly, we found that $P d \mathrm{C} 3 \mathrm{H} 17 \Delta \mathrm{COE}$ lines roughly showed wild type-like phenotypes (Figure 2), suggesting that overexpression of
PdC3H17 inhibits stem elongation likely depending on its $\mathrm{CCCH}$ domain.

\section{Different Drought Responses of $\mathrm{PdC} 3 \mathrm{H} 17$ and $P d C 3 H 17 \triangle C$ Overexpression Poplars Are Associated With the Changes of Their Stem Xylem Vessel Cell Number}

To determine the biological role of $\mathrm{PdC} 3 \mathrm{H} 17$ in drought stress, WT, $P d \mathrm{C} 3 \mathrm{H} 17 \mathrm{OE}$, and $P d C 3 H 17 \Delta \mathrm{COE}$ plants grown in soil for 3 months were exposed to drought treatment by withholding water. Most leaves of the WT poplars were seriously wilted on day 20, whereas those of $\mathrm{PaC} 3 \mathrm{H} 17 \mathrm{OE}$ plants remained normal (Figure 2D). The drought response of $P d C 3 H 17 \Delta C O E$ plants was slightly stronger than that of WT plants but significantly weaker than that of $P d \mathrm{C} 3 \mathrm{H} 17 \mathrm{OE}$ plants. Under the normally watered condition, WT and $P d C 3 H 17 \triangle \mathrm{COE}$ plants remained drastically faster growth than $\mathrm{PdC} 3 \mathrm{H} 17 \mathrm{OE}$ plants (Figure 2C).

We further investigated the effects of $\mathrm{PdC} 3 \mathrm{H} 17$ or $\mathrm{PdC} 3 \mathrm{H} 17 \Delta \mathrm{C}$ overexpression on alterations in physiology that may contribute to drought response. It is known that higher stem xylem water potential can prevent drought-induced hydraulic failure and enhance drought resistance (Choat et al., 2012). Our results indicated that stem xylem water potential was the highest in $P d \mathrm{C} 3 \mathrm{H} 17 \mathrm{OE}$ plants, followed in $P d \mathrm{C} 3 \mathrm{H} 17 \Delta \mathrm{C}$ OE plants, and the lowest in WT plants under drought stress (Figure 3A), which was consistent with their visible phenotypes (Figure 2D). By contrast, the three genotypes showed similar stem water potential under control condition (Figure 3A). We then analyzed the morphology of stem xylem cells. As indicated in Figures 3B, C the vessel number per unit of area was the greatest in $P d \mathrm{C} 3 \mathrm{H} 17 \mathrm{OE}$ plants, following in $\mathrm{PdC} 3 \mathrm{H} 17 \Delta \mathrm{COE}$ plants, and the smallest in WT plants. No significant difference for cell size of stem xylem vessels was observed between the three genotypes. It is possible that the increase of stem vessel cell number in $\mathrm{PdC} 3 \mathrm{H} 17$ and $P d \mathrm{C} 3 \mathrm{H} 17 \Delta \mathrm{C}$ overexpression poplars contributes to more effective water transport, thereby promoting drought tolerance.

\section{Photosynthetic Capacities Are Differentially Repressed in $\mathrm{PdC} 3 \mathrm{H17}$ and PdC3H17 $\triangle \mathrm{C}$ Overexpression Poplars After Drought Treatment}

Four photosynthetic parameters (chlorophyll $\mathrm{a}+\mathrm{b}$ content, stomatal conductance, net photosynthetic rate, and transpiration) were detected in WT, $P d C 3 H 17 \mathrm{OE}$, and $P d C 3 H 17 \triangle \mathrm{COE}$ poplars (Figure 4). At 20th day of normal-watered condition, chlorophyll $\mathrm{a}+\mathrm{b}$ content was roughly similar among three genotypes (Figure 4A). However, stomatal conductance, transpiration, and net photosynthetic rate in $\mathrm{PdC} 3 \mathrm{H} 17 \mathrm{OE}$ plants were markedly lower than those in WT and $P d C 3 H 17 \Delta \mathrm{COE}$ plants (Figures 4B-D). When treated by drought stress for 20 days, four photosynthetic parameters were decreased in all detected plants, but these decreases were more obvious in both WT and $P d C 3 H 17 \triangle \mathrm{COE}$ plants than in $P d \mathrm{C} 3 \mathrm{H} 17 \mathrm{OE}$ plants (Figure 4). These results suggest that overexpression of $\mathrm{PdC} 3 \mathrm{H} 17$ increases drought tolerance likely depending on its $\mathrm{CCCH}$ domain. 
A
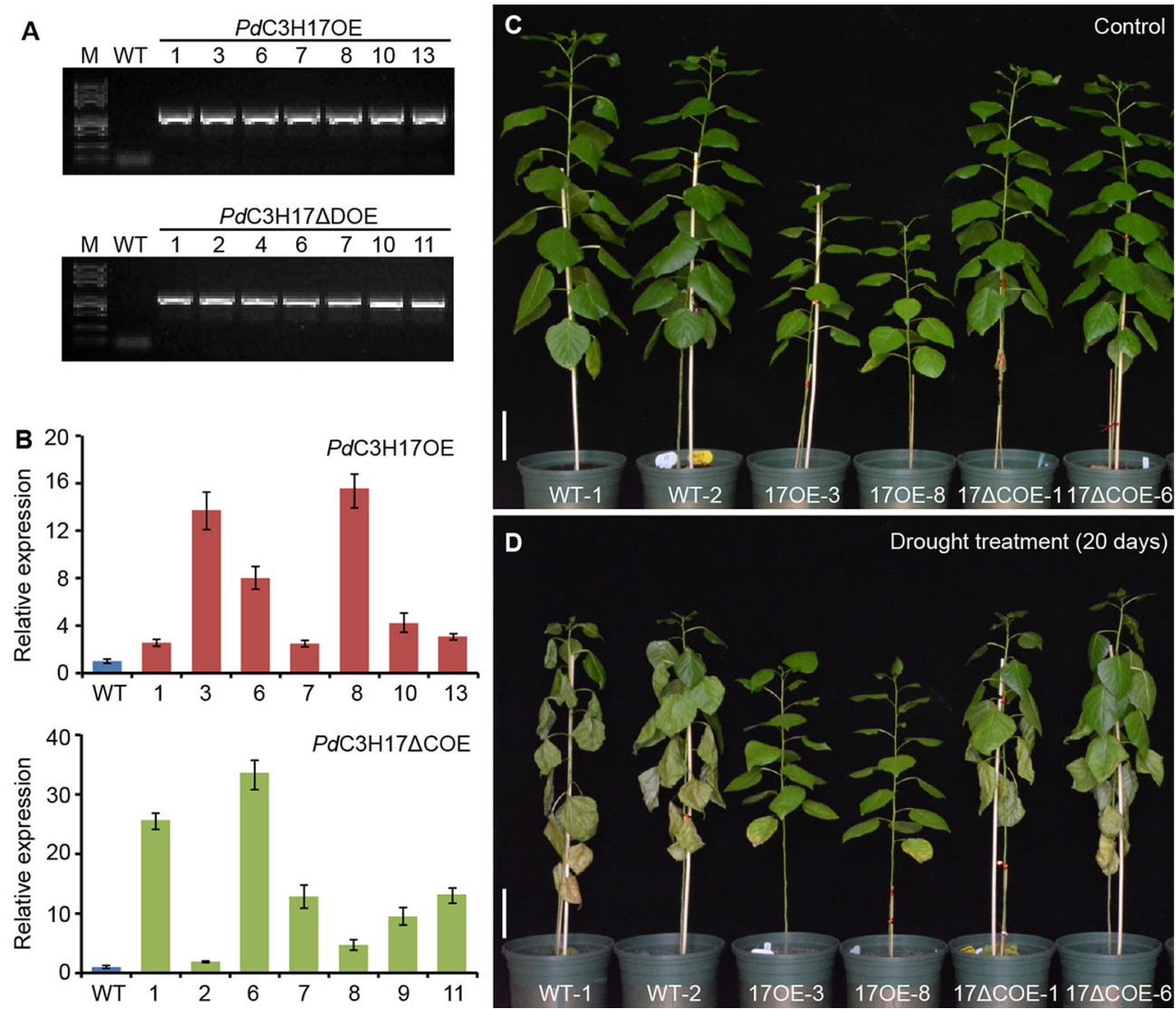

FIGURE 2 | Morphological differences between wild-type and transgenic poplars overexpressing PdC3H17 or PdC3H17 $\triangle \mathrm{C}$ after well-watered or drought stress conditions for 20 days. (A) PCR identification of representative PdC3H17 and PdC3H17 $A C$ overexpression poplar lines using pro35S and gene-specific primers. Wild-type (WT) plants were used as the control. (B) qRT-PCR analysis of PdC3H17 expression in WT, PdC3H17OE, or PdC3H17 $\Delta$ COE poplar lines. (C, D) Drought response of 3-month-old representative WT, PdC3H17OE (17OE), and PdC3H17 $\mathrm{COOE}(17 \Delta \mathrm{COE})$ poplar lines at the 20th day of well-watered (C) or drought (D) treatment. Bars $=10 \mathrm{~cm}$.

\section{The ROS-Scavenging Abilities Are Differentially Increased in $\mathrm{PdC} 3 \mathrm{H} 17$ and PdC3H17 $\triangle \mathrm{C}$ Overexpression Poplars After Drought Treatment}

ROS play a key role in the acclimation process of plants to drought stress (Choudhury et al., 2017). We thus examined whether overexpression of $\mathrm{PdC} 3 \mathrm{H} 17$ or $\mathrm{PdC} 3 \mathrm{H} 17 \Delta \mathrm{C}$ affects the accumulation of ROS in transgenic poplars. The levels of $\mathrm{H}_{2} \mathrm{O}_{2}$, a major ROS, were slightly higher in $\mathrm{PdC} 3 \mathrm{H} 17 \mathrm{OE}$ plants than in WT and $P d \mathrm{C} 3 \mathrm{H} 17 \triangle \mathrm{COE}$ plants under control condition. However, at 20 days of drought stress a lower accumulation of $\mathrm{H}_{2} \mathrm{O}_{2}$ was observed in $P d \mathrm{C} 3 \mathrm{H} 17 \mathrm{OE}$ plants relative to other two genotypes (Figure 5A). Further, the $P d \mathrm{C} 3 \mathrm{H} 17 \Delta \mathrm{COE}$ plants showed less increase of $\mathrm{H}_{2} \mathrm{O}_{2}$ level than WT plants. These results indicated that overexpression of $P d C 3 H 17$ and $P d C 3 H 17 \Delta C$ in poplar differentially affected droughtinduced ROS accumulation. Similarly, the increased levels of MDA, an indicator of cytomembrane oxidative damage (Shi et al., 2014), were the highest in WT plans and the lowest in $P d \mathrm{C} 3 \mathrm{H} 17 \mathrm{OE}$ plants after drought treatment (Figure 5B), suggesting that WT plants may undergo more serious membrane damage than these transgenic plants.

6APX, CAT, POD, and SOD are crucial antioxidants that can scavenge ROS (Choudhury et al., 2017). We further detected the activities of these four enzymes in drought-treated and control WT and transgenic poplars. Corresponded with the $\mathrm{H}_{2} \mathrm{O}_{2}$ levels, the activities of APX, CAT, POD, and SOD were most obviously elevated in $P d \mathrm{C} 3 \mathrm{H} 17 \mathrm{OE}$ plants, following $P d \mathrm{C} 3 \mathrm{H} 17 \Delta \mathrm{COE}$ plants, and less significantly increased in WT plants when treated by drought (Figures 5C-F). Besides, free proline, soluble protein, and soluble sugar contents were measured in these poplars (Figures 6A-C). Under control condition, $P d \mathrm{C} 3 \mathrm{H} 17 \mathrm{OE}$ plants showed higher levels of soluble protein and soluble sugar than WT and $P d \mathrm{C} 3 \mathrm{H} 17 \Delta \mathrm{COE}$ plants (Figures 6B-C). After drought stress for 20 days, free proline, soluble protein, and soluble sugar were more obviously accumulated in $\mathrm{Pd} \mathrm{C} 3 \mathrm{H} 17 \mathrm{OE}$ plants than in other two genotypes (Figures $\mathbf{6 A - C}$ ). These data indicated that PdC3H17 overexpression may enhance the ROS scavenging capacity and thereby confer plant resistance to drought stress. 

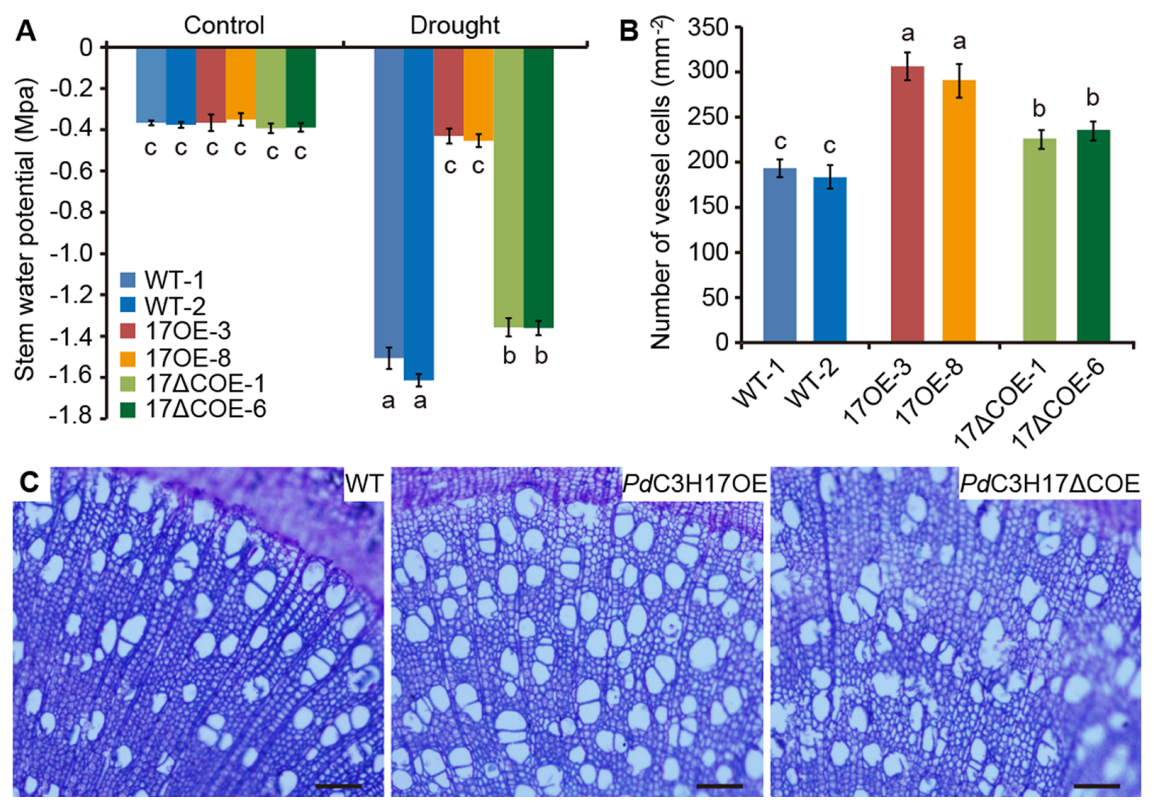

FIGURE 3 | Stem water potential and xylem vessel cell numbers of WT, PdC3H17OE, and PdC3H17 $\triangle \mathrm{COE}$ poplars. (A) Statistical analysis of stem water potential of 4-month-old WT, PdC3H17OE (17OE), and PdC3H17 $\mathrm{COE}$ (17 $\triangle \mathrm{COE})$ poplars under well-watered and drought stress conditions for 20 days. (B) Statistical analysis of the numbers of stem xylem vessel cells in 3-month-old WT, 17OE, and 17 $\mathrm{COOE}$ poplars. At least six plants from two lines in each genotype were selected for measurement of stem water potential and xylem vessel cells. Data are presented as mean \pm SD. Different letters above bars denote statistical significance between treatments of samples $(P<0.05)$. (C) Basal stem sections of 3-month-old representative WT, $P d C 3 H 17 \mathrm{OE}$, and PdC3H17 $\mathrm{CCOE}$ plants. Bars $=100 \mu \mathrm{m}$.
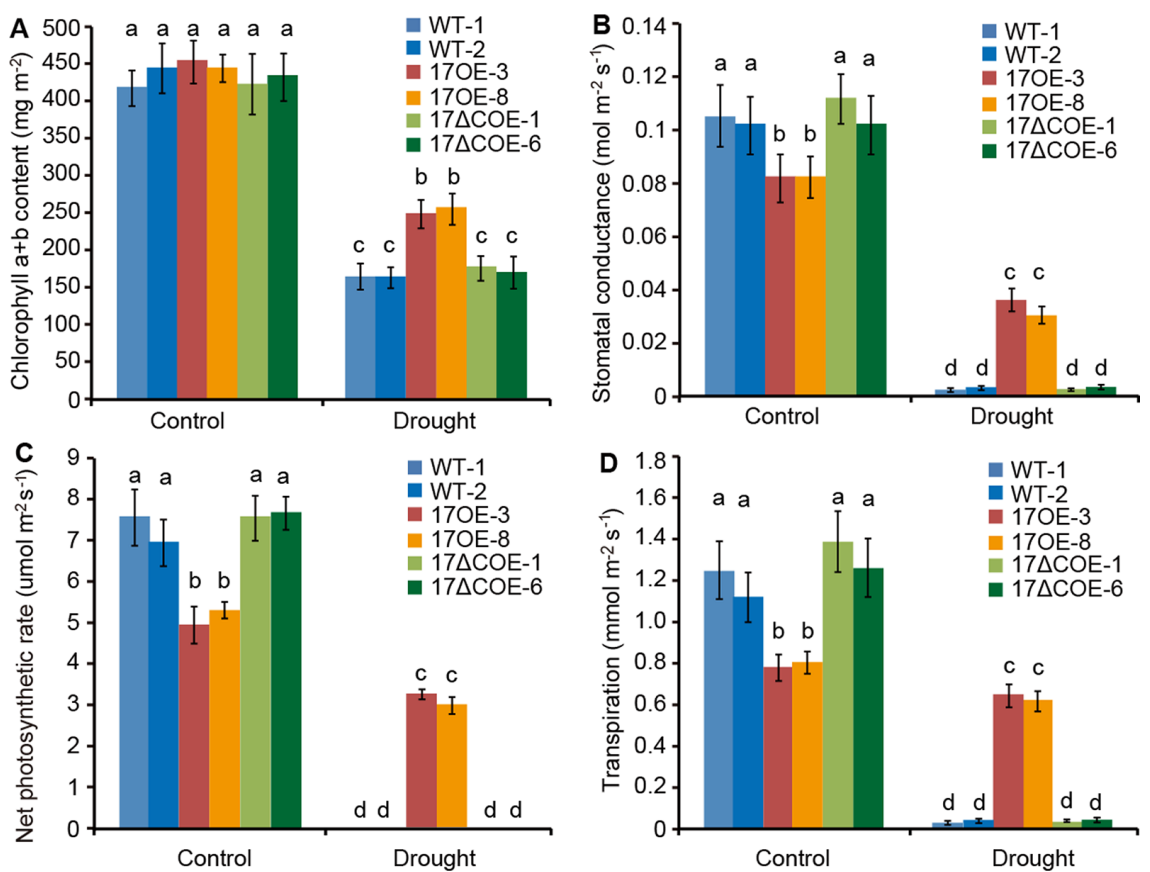

FIGURE 4 | Variation in photosynthetic parameters of 3-month-old PdC3H17OE (17OE) and PdC3H17 $\triangle \mathrm{COE}$ (17 $\mathrm{COOE})$ plants relative to those of WT plants after well-watered or drought stress conditions for 20 days. (A) Chlorophyll a $+b$ content. (B) Stomatal conductance. (C) Net photosynthetic rate. (D) Transpiration. At least six plants of two lines in each genotype were measured. Data are presented as mean $\pm \mathrm{SD}$. Different letters above bars denote statistical significance between treatments of leaves $(P<0.05)$. 

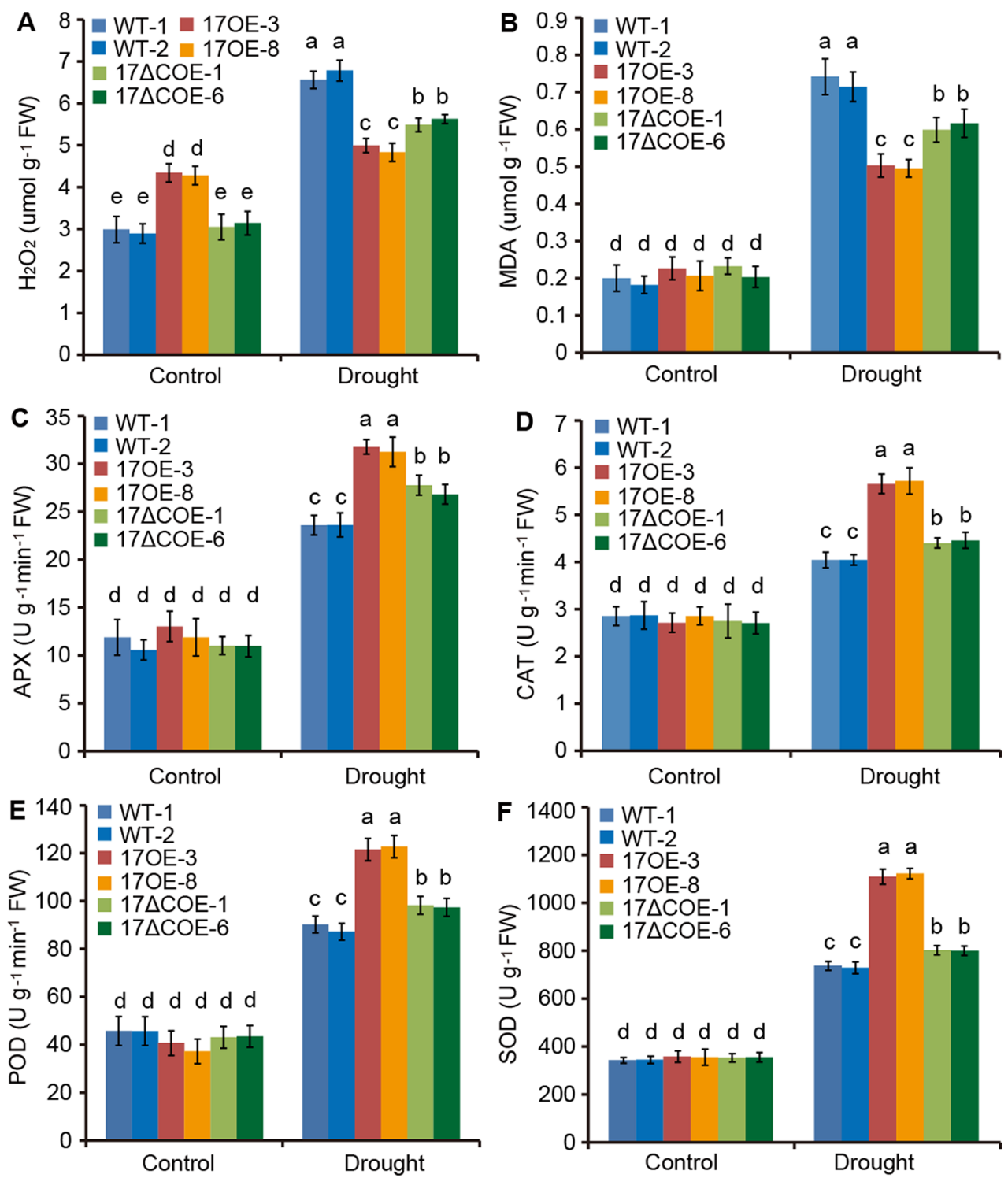

FIGURE 5 | Quantitative analyses of $\mathrm{H}_{2} \mathrm{O}_{2}$ and four antioxidants in 3-month-old WT, PdC3H17OE (17OE), and PdC3H17 $\triangle \mathrm{COE}$ (17 $\Delta \mathrm{COE}$ ) poplars after well-watered or drought stress conditions for 20 days. (A) $\mathrm{H}_{2} \mathrm{O}_{2}$, hydrogen peroxide. (B) MDA, malondialdehyde. (C) APX, ascorbate peroxidase. (D) CAT, catalase. (E) POD, peroxidase. (F) SOD, superoxide dismutase. At least six plants of two lines in each genotype were measured. Data are presented as mean \pm SD. Different letters above bars denote statistical significance between treatments of leaves $(P<0.05)$.

\section{Weaker Growth Inhibition is Observed in PdC3H17 Overexpression Poplars During Drought Stress}

We further investigate whether overexpression of $\mathrm{PdC} 3 \mathrm{H} 17$ or $P d C 3 H 17 \Delta C$ in poplar affects stem growth in the absence of water stress. Statistical analyses revealed that drought treatment resulted in a significantly greater decrease of both stem elongation and thickening rates in $\mathrm{WT}$ and $P d \mathrm{C} 3 \mathrm{H} 17 \Delta \mathrm{COE}$ poplars than in $P d \mathrm{C} 3 \mathrm{H} 17 \mathrm{OE}$ poplars compared to control condition (Figure 7). Furthermore, these decreases were more obvious in WT plants than in $\mathrm{PdC} 3 \mathrm{H} 17 \Delta \mathrm{COE}$ plants. Therefore,
PdC3H17OE poplars maintained higher growth than WT plants under long-term water deficit conditions.

\section{DISCUSSION}

Drought is the primary abiotic stress responsible for inhibiting poplar growth (Yin et al., 2004; Monclus et al., 2006; Harfouche et al., 2014). Clarifying the regulatory mechanisms of drought tolerance in poplar is essential for ecological conservation and wood production. In this study, we provide evidence showing 

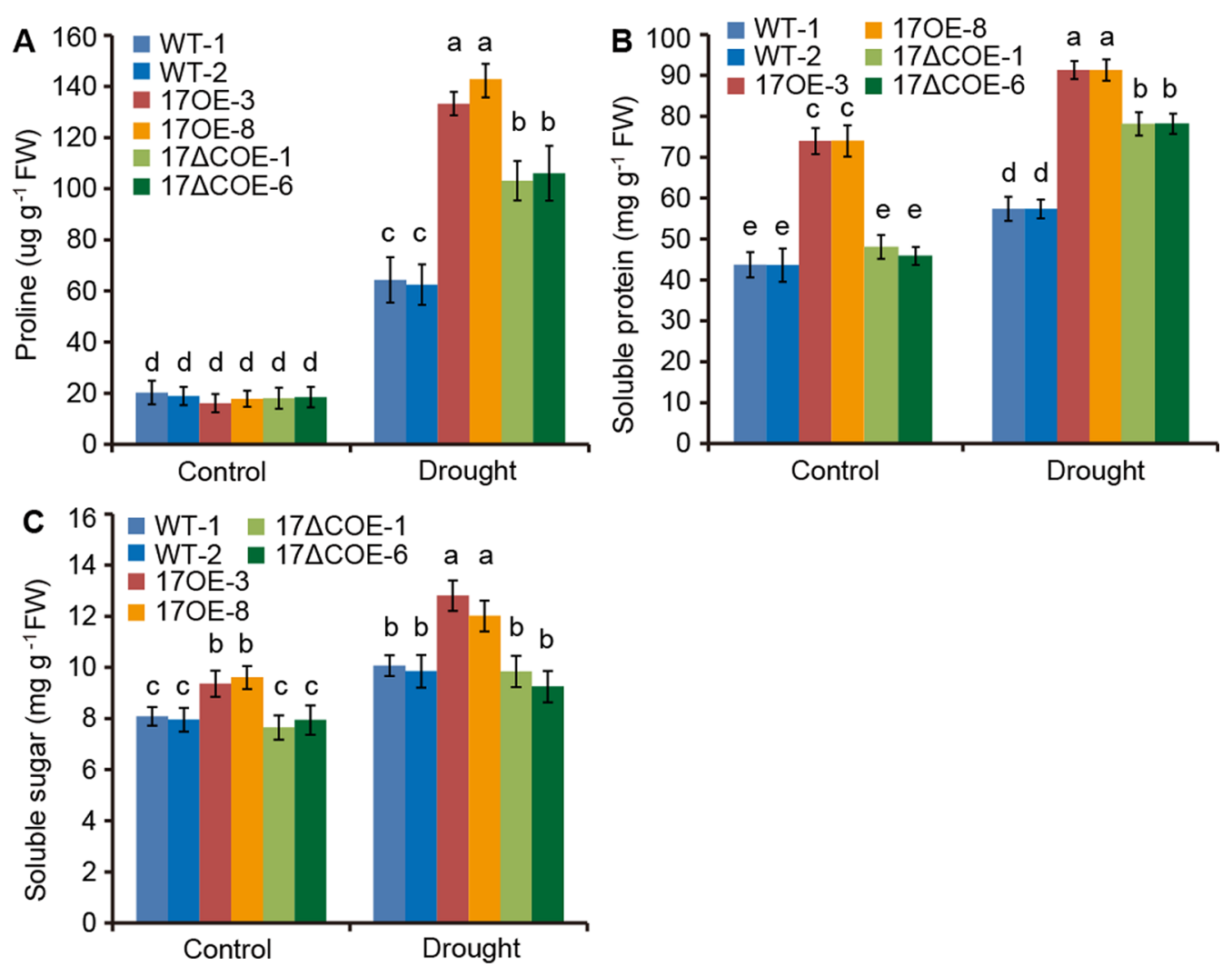

FIGURE 6 | Quantitative analyses of osmotic adjustment substances in 3-month-old WT, PdC3H17OE (17OE), and PdC3H17 $\triangle \mathrm{COE}$ (17 $\triangle \mathrm{COE})$ poplars after wellwatered or drought stress conditions for 20 days. (A) Free proline. (B) Soluble protein. (C) Soluble sugar. At least six plants of two lines in each genotype were measured. Data are presented as mean \pm SD. Different letters above bars denote statistical significance between treatments of leaves $(P<0.05)$. $P<0.05$.

that $\mathrm{PdC} 3 \mathrm{H} 17$ is a novel regulator of drought response and tolerance. Overexpression of $\mathrm{PdC} 3 \mathrm{H} 17$ in a hybrid poplar resulted in a significant increase in drought tolerance in comparison with WT plants, correlating with visible alterations of their physiological parameters. As far as we know, this is the first report of CCCHmediated drought response in woody species.

Wood is made up of xylem, the conductive tissue that transports water from soil to leaves and provides mechanical support for the entire plant (Evert, 2006). Hydraulic conductivity in xylem is highly related to cell size and number of stem xylem vessels (Fisher et al., 2007; Li et al., 2019). For instance, transgenic poplars overexpressing PtrNAC006, PtrNAC007, or PtrNAC120 have smaller vessel lumen area and more vessel cells than WT plants, resulting in stronger drought tolerance (Li et al., 2019). Here, our observations of stem sections revealed that the $P d \mathrm{C} 3 \mathrm{H} 17 \mathrm{OE}$ poplars had more xylem vessel cells than WT controls, correlating with higher stem water potential in these transgenic plants (Figure 3). These may result in stronger stem hydraulic conductance in $P d \mathrm{C} 3 \mathrm{H} 17 \mathrm{OE}$ plants than in WT plants. Stress-resistant plants often have a dwarf form ( $\mathrm{Hu}$ et al., 2005; Yu et al., 2008). Because the dwarf is benefit for reducing water and energy consumption and facilitates energy redistribution. Our current results revealed that non-stressed PdC3H17OE poplars showed growth inhibition phenotypes with reduced plant height and decreased stomatal conductance, transpiration, and net photosynthetic rate (Figures 2 and 4). Further, $P d \mathrm{C} 3 \mathrm{H} 17 \mathrm{OE}$ lines showed higher stem elongation and thickening rates than WT plants under water-deficit condition (Figure 7). Therefore, $P d \mathrm{C} 3 \mathrm{H} 17 \mathrm{OE}$ poplars enhance drought tolerance likely due to greater hydraulic conductance and dwarf phenotype.

$\mathrm{H}_{2} \mathrm{O}_{2}$ is one of the major ROS in plants, and its level is significantly increased under stress conditions (Bhattacharjee, 2012; Choudhury et al., 2017). To minimize oxidative damage, plants have developed sophisticated ROS-scavenging mechanisms. Here, we found that drought stress led to mass accumulation of $\mathrm{H}_{2} \mathrm{O}_{2}$ in all detected poplars (Figure 5A). However, the accumulation of $\mathrm{H}_{2} \mathrm{O}_{2}$ in WT plants was obviously higher than that in $\mathrm{PdC} 3 \mathrm{H} 17 \mathrm{OE}$ plants (Figure 5A), and meanwhile the stomatal conductance and photosynthesis in the WT lines almost reached zero (Figures 4B, C). Accordingly, the activities of four major ROS-scavenging enzymes (APX, CAT, POD, and SOD) were increased less significantly in WT plants than in $P d \mathrm{C} 3 \mathrm{H} 17 \mathrm{OE}$ plants after drought treatment (Figures 5C-F). Thus, another possible reason for strong drought tolerance of $P d \mathrm{C} 3 \mathrm{H} 17 \mathrm{OE}$ poplars may be more effective activation of the antioxidant system.

Tristetraprolin (hTTP) and AtC3H14 are the orthologs of $P d \mathrm{C} 3 \mathrm{H} 17$ in human and Arabidopsis, respectively 


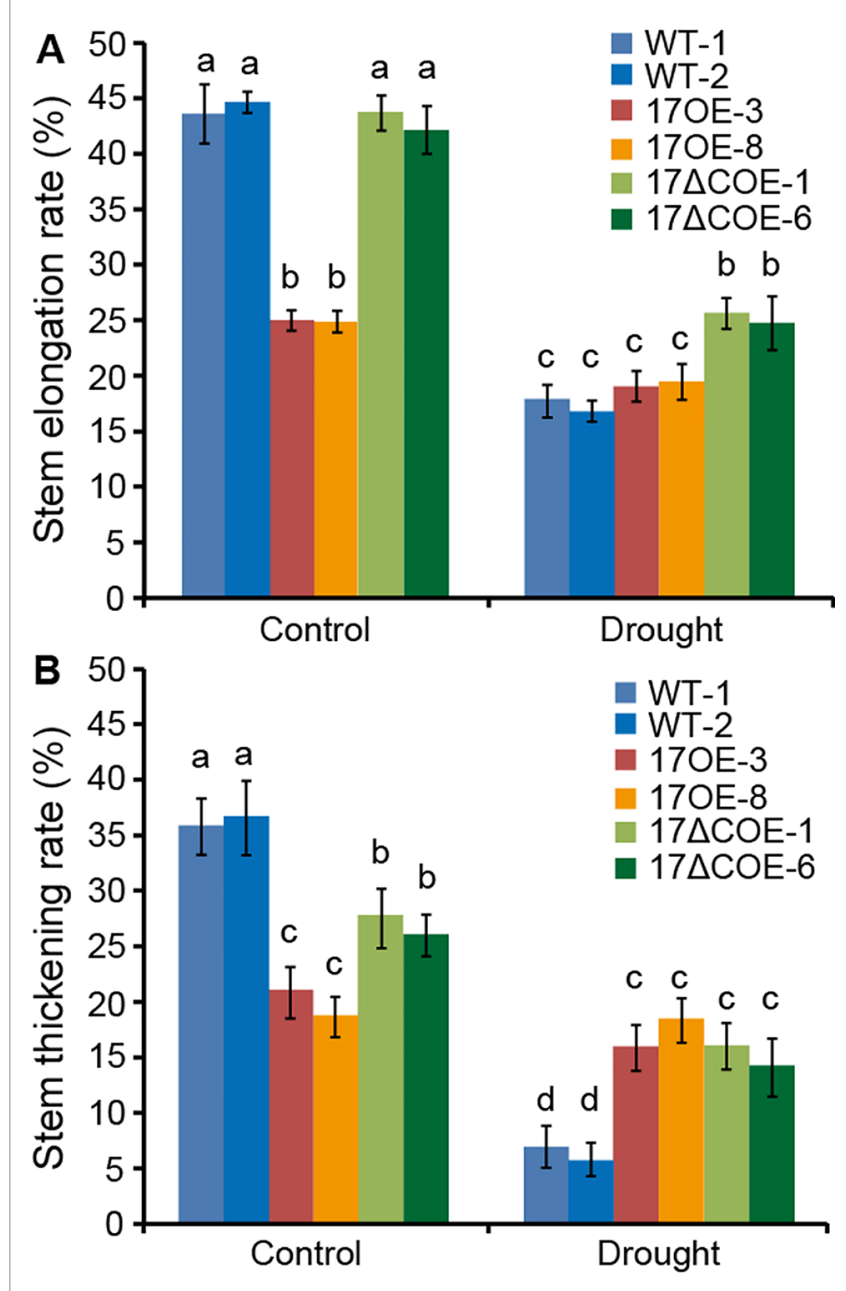

FIGURE 7 | Stem elongation and thickening rates of 3-month-old WT, PdC3H17OE (17OE), and PdC3H17 $\triangle \mathrm{COE}$ (17 $\mathrm{COOE})$ poplars after wellwatered or drought stress conditions for 20 days. (A) Stem elongation rate. (B) Stem thickening rate. At least six plants of two lines in each genotype were measured. Data are presented as mean \pm Different letters above bars denote statistical significance between treatments of samples $(P<0.05)$.

(Chai et al., 2012). The two proteins have the DNA/RNA binding abilities and may perform transcriptional regulation in the nucleus and RNA regulation in cytoplasm (Lai et al., 1999; Kim et al., 2014; Chai et al., 2015). The best-known CCCH protein is human hTTP, which binds to AU-rich elements (AREs; AUUUA) in the 3'untranslated region (UTR) of target genes such as TNF-alpha, and performs post-transcriptional regulation (Lai et al., 1999). Further, the CCCH domain of hTTP is necessary for the deadenylation and degradation of target mRNAs in cytoplasmic foci (Lai et al., 1999; Michel et al., 2003). RNA-EMSA data showed that AtC3H14 from Arabidopsis also requires its $\mathrm{CCCH}$ domain for target RNA binding (Kim et al., 2014). Our current results revealed that $P d \mathrm{C} 3 \mathrm{H} 17 \mathrm{OE}$ poplars exhibited dwarf and drought-tolerance phenotypes whereas morphology and drought response of PdC $3 \mathrm{H} 17 \Delta \mathrm{COE}$ poplars are more similar to WT plants (Figure 2), suggesting that overexpression of $\mathrm{PdC} 3 \mathrm{H} 17$ in a hybrid poplar may confer drought tolerance and inhibit stem elongation depending of its $\mathrm{CCCH}$ domain. Considering that $P d \mathrm{C} 3 \mathrm{H} 17$, like hTTP and AtC3H14, shares typical $\mathrm{CX}_{8} \mathrm{CX}_{5} \mathrm{CX}_{3} \mathrm{H}$ motifs and is targeted to cytoplasmic foci (Chai et al., 2014), we speculate that $\mathrm{PdC} 3 \mathrm{H} 17$ might function in drought tolerance and stem elongation through $\mathrm{CCCH}$ domaindependent and post-transcriptional regulation in Populus. Further studies need to be conducted to resolve these ambiguities. Interestingly, we found that the $\mathrm{N}$-terminal sequence of $P d \mathrm{C} 3 \mathrm{H} 17$, but not full-length $P d \mathrm{C} 3 \mathrm{H} 17$, had transcriptional activation ability in yeast cells (Figure 1). LIC, a CCCH protein in rice, is shown to function as a negative regulator of the Brassinosteroid (BR) signaling pathway through direct suppression of BZR1 targets (Zhang et al., 2012). Thus, it is possible that $P d \mathrm{C} 3 \mathrm{H} 17$, like $\mathrm{LIC}$, acts as a transcriptional repressor of stem development and stress resistance, in addition to functioning at the post-transcriptional level. This hypothesis needs to be further validated experimentally.

In conclusion, we developed transgenic $\mathrm{PdC} 3 \mathrm{H} 17$ - or $P d C 3 H 17 \Delta C$-expressing poplar plants. $P d \mathrm{C} 3 \mathrm{H} 17 \mathrm{OE}$ poplar was slightly dwarf, had more stem xylem vessel cells, and enhanced ROS-scavenging abilities, thereby reinforcing plant tolerance to drought stress. In contrast, $P d \mathrm{C} 3 \mathrm{H} 17 \Delta \mathrm{COE}$ poplar showed WTlike phenotypes, indicating the requirement of its $\mathrm{CCCH}$ domain for drought response. Our previous study demonstrated that $P d \mathrm{C} 3 \mathrm{H} 17 \mathrm{OE}$ poplars had significantly wider xylem and thicker secondary cell walls than WT controls after growth of 4 months (Chai et al., 2014). Therefore, $P d \mathrm{C} 3 \mathrm{H} 17$ may have potential for use in the genetic improvement of drought tolerance and wood production in Populus.

\section{DATA AVAILABILITY STATEMENT}

The datasets generated for this study are available on request to the corresponding authors.

\section{AUTHOR CONTRIBUTIONS}

GC designed the experiment, performed data processing, and drafted the manuscript. YZhu, CW, YZha, SC, DW, and QL prepared the materials and performed the experiments. GZ conceived the study and revised the manuscript. All authors read and approved the final version of the manuscript.

\section{FUNDING}

Financial support for this work was obtained from National Key Scientific Research Project of China (2016YFD0600104), National Key Program on Transgenic Research (2018ZX08020002), National Natural Science Foundation of China (31670606, 31570670, 31770315, and 31700526), Major Basic Research Project of Shandong Natural Science Foundation (ZR2018ZC0335), Shandong Provincial Natural Science Foundation (ZR2017BC096, ZR2017BC078, and ZR2019BC006), and Taishan Scholar Program of Shandong (to GZ). 


\section{REFERENCES}

Bhattacharjee, S. (2012). The language of reactive oxygen species signaling in plants. J. Bot. 2012, 1-22. doi: 10.1155/2012/985298

Bogamuwa, S. P., and Jang, J. C. (2014). Tandem CCCH zinc finger proteins in plant growth, development and stress response. Plant Cell Physiol. 55, 13671375. doi: $10.1093 / \mathrm{pcp} / \mathrm{pcu} 074$

Chai, G., Hu, R., Zhang, D., Qi, G., Zuo, R., Cao, Y., et al. (2012). Comprehensive analysis of $\mathrm{CCCH}$ zinc finger family in poplar (Populus trichocarpa). BMC Genomics 13, 253. doi: 10.1186/1471-2164-13-253

Chai, G., Qi, G., Cao, Y., Wang, Z., Yu, L., Tang, X., et al. (2014). Poplar PdC3H17 and $P d \mathrm{C} 3 \mathrm{H} 18$ are direct targets of $P d \mathrm{MYB} 3$ and $P d \mathrm{MYB} 21$, and positively regulate secondary wall formation in Arabidopsis and poplar. New Phytol. 203 (2), 520-534. doi: 10.1111/nph.12825

Chai, G., Kong, Y., Zhu, M., Yu, L., Qi, G., Tang, X., et al. (2015). Arabidopsis $\mathrm{C} 3 \mathrm{H} 14$ and $\mathrm{C} 3 \mathrm{H} 15$ have overlapping roles in the regulation of secondary wall thickening and anther development. J. Exp. Bot. 66, 2595-2609. doi: 10.1093/ jxb/erv060

Choat, B., Jansen, S., Brodribb, T. J., Cochard, H., Delzon, S., Bhaskar, R., et al. (2012). Global convergence in the vulnerability of forests to drought. Nature 491, 752-755. doi: 10.1038/nature11688

Choudhury, F. K., Rivero, R. M., Blumwald, E., and Mittler, R. (2017). Reactive oxygen species, abiotic stress and stress combination. Plant J. 90 (5), 856-867. doi: $10.1111 /$ tpj.13299

$\mathrm{Du}$, J., and Groover, A. (2010). Transcriptional regulation of secondary growth and wood formation. J. Integer. Plant Biol. 52 (1), 17-27. doi: 10.1111/j.17447909.2010.00901.x

Evert, R. F. (2006). Esau's plant anatomy: meristems, cells, and tissues of the plant body: their structure, function, and development (Hoboken, NJ: John Wiley \& Sons).

Fisher, J. B., Goldstein, G., Jones, T. J., and Cordell, S. (2007). Wood vessel diameter is related to elevation and genotype in the Hawaiian tree Metrosideros polymorpha (Myrtaceae). Am. J. Bot. 94 (5), 709-715. doi: 10.3732/ajb.94.5.709

Harfouche, A., Meilan, R., and Altman, A. (2014). Molecular and physiological responses to abiotic stress in forest trees and their relevance to tree improvement. Tree Physiol. 34 (11), 1181-1198. doi: 10.1093/treephys/tpu012

He, F., Wang, H. L., Li, H. G., Su, Y., Li, S., Yang, Y., et al. (2018). PeCHYR1, a ubiquitin E3 ligase from Populus euphratica, enhances drought tolerance via ABA-induced stomatal closure by ROS production in Populus. Plant Biotechnol. J. 16 (8), 1514-1528. doi: 10.1111/pbi.12893

Hu, L., Lu, H., Liu, Q., Chen, X., and Jiang, X. (2005). Overexpression of $m t l D$ gene in transgenic Populus tomentosa improves salt tolerance through accumulation of mannitol. Tree Physiol. 25 (10), 1273-1281. doi: 10.1093/treephys/25.10.1273

Jan, A., Maruyama, K., Todaka, D., Kidokoro, S., Abo, M., Yoshimura, E., et al. (2013). OsTZF1, a CCCH-tandem zinc finger protein, confers delayed senescence and stress tolerance in rice by regulating stress-related genes. Plant Physiol. 161, 1202-1216. doi: 10.1104/pp.112.205385

Kim, W. C., Kim, J. Y., Ko, J. H., Kang, H., Kim, J., and Han, K. H. (2014). AtC3H14, a plant-specific tandem CCCH zinc-finger protein, binds to its target mRNAs in a sequence-specific manner and affects cell elongation in Arabidopsis thaliana. Plant J. 80, 772-784. doi: 10.1111/tpj.12667

Lai, W. S., Carballo, E., Strum, J. R., Kennington, E. A., Phillips, R. S., and Blackshear, P. J. (1999). Evidence that tristetraprolin binds to AU-rich elements and promotes the deadenylation and destabilization of tumor necrosis factor alpha mRNA. Mol. Cell. Biol. 19 (6), 4311-4323. doi: 10.1128/mcb.19.6.4311

Lee, S. J., Jung, H. J., Kang, H., and Kim, S. Y. (2012). Arabidopsis zinc finger proteins AtC3H49/AtTZF3 and AtC3H20/AtTZF2 are involved in ABA and JA responses. Plant Cell Physiol. 53, 673-686. doi: 10.1093/pcp/pcs023

Li, S., Lin, Y. J., Wang, P., Zhang, B., Li, M., Chen, S., et al. (2019). The AREB1 transcription factor influences histone acetylation to regulate drought responses and tolerance in Populus trichocarpa. Plant Cell. 31 (3), 663-686. doi: $10.1105 /$ tpc. 18.00437
Lin, P. C., Pomeranz, M. C., Jikumaru, Y., Kang, S. G., Hah, C., Fujioka, S., et al. (2011). The Arabidopsis tandem zinc finger protein AtTZF1 affects ABA- and GA-mediated growth, stress and gene expression responses. Plant J. 65, $253-$ 268. doi: 10.1111/j.1365-313X.2010.04419.x

Livak, K. J., and Schmittgen, T. D. (2001). Analysis of relative gene expression data using real-time quantitative PCR and the 2 (-Delta Delta C (T)) method. Methods 25, 402-408. doi: 10.1006/meth.2001.1262

Michel, S. L. J., Guerrerio, A. L., and Berg, J. M. (2003). Selective RNA binding by a single CCCH zinc-binding domain from Nup475 (Tristetraprolin). Biochemistry 42 (16), 4626-4630. doi: 10.1021/bi034073h

Monclus, R., Dreyer, E., Villar, M., Delmotte, F. M., Delay, D., Petit, J. M., et al. (2006). Impact of drought on productivity and water use efficiency in genotypes of Populus deltoides x Populus nigra. New Phytol. 169 (4), $765-$ 777. doi: $10.1111 /$ j.1469-8137.2005.01630.x

Shi, H., Ye, T., Zhu, J. K., and Chan, Z. (2014). Constitutive production of nitric oxide leads to enhanced drought stress resistance and extensive transcriptional reprogramming in Arabidopsis. J. Exp. Bot. 65 (15), 4119-4131. doi: 10.1093/ jxb/eru184

Wang, D., Guo, Y. H., Wu, C. G., Yang, G. D., Li, Y. Y., and Zheng, C. C. (2008). Genome-wide analysis of $\mathrm{CCCH}$ zinc finger family in Arabidopsis and rice. BMC Genomics 9, 44. doi: 10.1186/1471-2164-9-44

Wang, W., Liu, B., Xu, M., Jamil, M., and Wang, G. (2015). ABA-induced CCCH tandem zinc finger protein $\mathrm{Os} C 3 \mathrm{H} 47$ decreases $\mathrm{ABA}$ sensitivity and promotes drought tolerance in Oryza sativa. Biochem. Biophys. Res. Commun. 464, 3337. doi: 10.1016/j.bbrc.2015.05.087

Wang, C., Liu, S., Dong, Y., Zhao, Y., Geng, A., Xia, X., et al. (2016). PdEPF1 regulates water-use efficiency and drought tolerance by modulating stomatal density in poplar. Plant Biotechnol. J. 14 (3), 849-860. doi: 10.1111/pbi.12434

Ye, Z. H., and Zhong, R. (2015). Molecular control of wood formation in trees. J. Exp. Bot. 66, 4119-4131. doi: 10.1093/jxb/erv081

Yin, C. Y., Duan, B. L., Wang, X., and Li, C. Y. (2004). Morphological and physiological responses of two contrasting poplar species to drought stress and exogenous abscisic acid application. Plant Sci. 167, 1091-1097. doi: 10.1016/ j.plantsci.2004.06.005

Yu, H., Chen, X., Hong, Y., Wang, Y., Xu, P., Ke, S., et al. (2008). Activated expression of an Arabidopsis HD-START protein confers drought tolerance with improved root system and reduced stomatal density. Plant Cell. 20 (4), 1134-1151. doi: $10.1105 /$ tpc. 108.058263

Zhang, C., Xu, Y., Guo, S., Zhu, J., Huan, Q., Liu, H., et al. (2012). Dynamics of brassinosteroid response modulated by negative regulator LIC in rice. PloS Genet. 8, e1002686. doi: 10.1371/journal.pgen.1002686

Zhang, H., Gao, X., Zhi, Y., Li, X., Zhang, Q., Niu, J., et al. (2019). A non-tandem $\mathrm{CCCH}$-type zinc-finger protein, $\mathrm{IbC} 3 \mathrm{H} 18$, functions as a nuclear transcriptional activator and enhances abiotic stress tolerance in sweet potato. New Phytol. 223, 1918-1936. doi: 10.1111/nph.15925

Zhou, T., Yang, X., Wang, L., Xu, J., and Zhang, X. (2014). GhTZF1 regulates drought stress responses and delays leaf senescence by inhibiting reactive oxygen species accumulation in transgenic Arabidopsis. Plant Mol. Biol. 85, 163-177. doi: 10.1007/s11103-014-0175-z

Conflict of Interest: The authors declare that the research was conducted in the absence of any commercial or financial relationships that could be construed as a potential conflict of interest.

Copyright () 2020 Zhuang, Wang, Zhang, Chen, Wang, Liu, Zhou and Chai. This is an open-access article distributed under the terms of the Creative Commons Attribution License (CC BY). The use, distribution or reproduction in other forums is permitted, provided the original author(s) and the copyright owner(s) are credited and that the original publication in this journal is cited, in accordance with accepted academic practice. No use, distribution or reproduction is permitted which does not comply with these terms. 